\title{
A Multi-flow Multi-modal Assignment Procedure on Large Freight Transportation Networks
}

\author{
Bart JOURQUIN*
}

\begin{abstract}
Multi-Modal freight models are traditionally built following the well known "four steps model" in which generation, distribution, modal-split and assignment are seen as separated modules. An alternative approach is to represent the multi-modal network by means of a "monomodal" one, in which each particular transport operation (loading or unloading operation, transhipment, ...) is represented by a dedicated "virtual link".

This approach, promoted by several authors, often referenced to as "super networks" or "virtual networks" is proven to give interesting results, but has some kind of "hidden trap", linked to transport distances, that will be presented in this paper and that can only be solved using appropriate assignment techniques.

In a previous paper by Jourquin and Limbourg [15], it appeared that the equilibrium assignment techniques only partially solve the "distance trap", because the obtained solutions are very close to those obtained by a simple "all-or-nothing" assignment. This can mainly be explained by the fact that, in inter-urban, inter-regional or international traffic, only a small part of the journey is done during the peak-hours in congested areas, and that the demand data that can be collected on large areas is often available only on a yearly basis, making it practically impossible to have a good estimate of the demand at a given period of the day.

However, one can not consider that all the traffic between a given pair of origin and destination will be charged on the same transport mode and/or route. This paper outlines a new usable deterministic multi-flow/multi-modal assignment technique that can be applied on large (virtual) freight transportation networks and that offers a satisfactory solution to the "distance trap".
\end{abstract}

\section{Introduction}

A simple geographic network does not provide an adequate basis for detailed analyses of transport operations, as the same infrastructure can often be used in different ways. Thus, there is a need for a better modelling of the functions assumed by nodes, i.e. terminals and transhipment platforms, because the costs of the operations performed at these nodes are important in the total cost of transport. Indeed, a geographical multimodal transport network is not only made of links like roads, railways or waterways, on which vehicles move but also of connecting infrastructures like terminals or logistics platforms that exist at the nodes. To analyse transport operations over the network, costs or weights must be attached to the links over which goods are transported as well as to the connecting points where goods are handled. However, most of these transport or handling infrastructures can be used in different ways and at different costs. For example, boats of different sizes and operating costs can use the same waterway; at a terminal a truck's load can be transhipped on a train,

\footnotetext{
* Catholic University of Mons (FUCaM), Group Transport \& Mobility (GTM) 151 Chaussée de Binche, B-7000, Mons, Belgium

E-mail : bart.jourquin@fucam.ac.be
} 
bundled with some others on a boat or simply unloaded as it reaches its final destination. Normally, the costs of these alternative operations are different. In order to model this, one of the solutions is to represent each kind of operation in a node as a specific link of a "virtual network", for which a relevant cost is then computed.

This approach is proven to give interesting results, but has the drawback to generate much larger networks than the pure geographic representation of the studied area. Moreover, it has some kind of "hidden trap" which will be discussed in this paper, and that can be avoided when appropriate assignment techniques are used.

Different equilibrium assignment procedures where already described and tested on virtual networks (Jourquin and Limbourg [15]). The relevance of their application to inter-urban, inter-regional and international freight flows raises a number of questions that will be summarized in this paper.

An alternative, multi-flow assignment procedure, that solves the problems raised by the traditional equilibrium techniques and gives a satisfactory solution to the hidden "distance trap", will finally be described, discussed and illustrated.

\section{Virtual networks as an alternative network representation}

A simple geographic network does not provide an adequate basis for detailed analyses of transport operations as the same infrastructure, link or node, can be used in different ways. To solve this problem, the basic idea, initially proposed by Harker [9] and Crainic et al. [4], is to create a virtual link with specific costs for a particular use of an infrastructure. The concept of "supernetworks" proposed by Sheffi [18], that proposed "transfer" links between modal networks also provides a somewhat similar framework. The concept was systematised and implemented in a software package (Nodus) by Jourquin [13] and Jourquin \& Beuthe [14], permitting to apply the methodology to extensive multimodal networks.

This class of methodologies can be explained in an intuitive way by using the example of a simple multi-modal network, as shown in Figure 1. This network contains 4 nodes (1 to 4$)$ and 5 links $(a, b, c, d, e)$. The " $W$ " links represent waterways and the " $R$ " links rail tracks. The numbers after these letters correspond to the transportation means that can be used on the links. So, " $W 1$ " represents a waterway that can only support small barges and " $W 2$ " a waterway that can be used by both small and large barges.

To go from node " $a$ " to " $d$ ", it could be that route through links $1+3$ and using large barges and trains is less expensive than route 5, using exclusively small barges. Indeed, computing costs and routes on this kind of networks is not immediate :

a) Different costs can be assigned on a single link, depending on which transportation means is used. In this example, the use of a small barge on link 1 has a different cost than the use of a large barge on the same link;

b) The same is true for the nodes: in the given example, the simple transit of a small barge from link 1 to link 2 can be done at no cost, but the transhipment from a large barge onto a train that will go on link 3 represents an important cost.

This problem can be usefully handled on the corresponding virtual network illustrated in Figure 2, provided that the relevant costs are attached to each of the virtual links. In this 


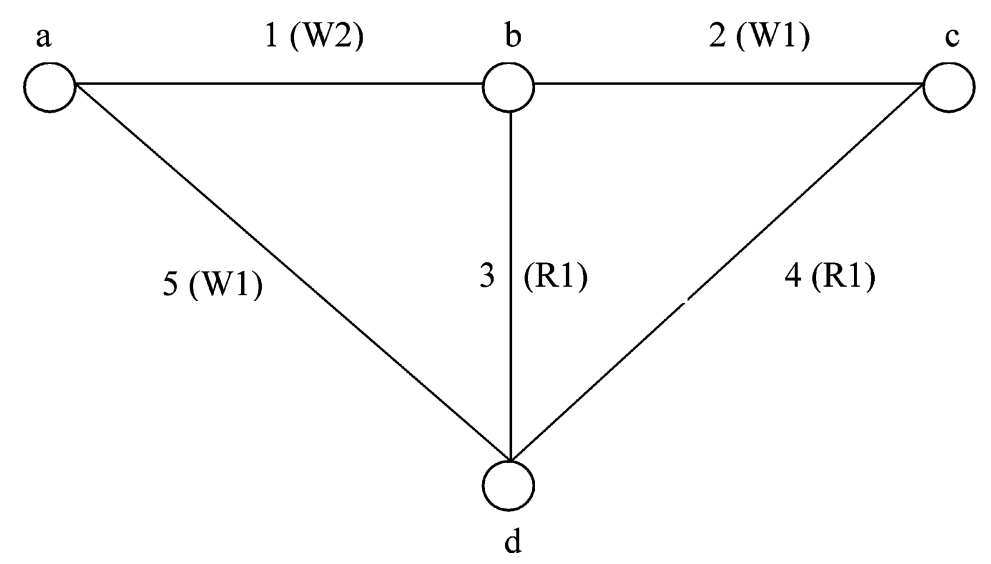

Figure 1: Multimodal network

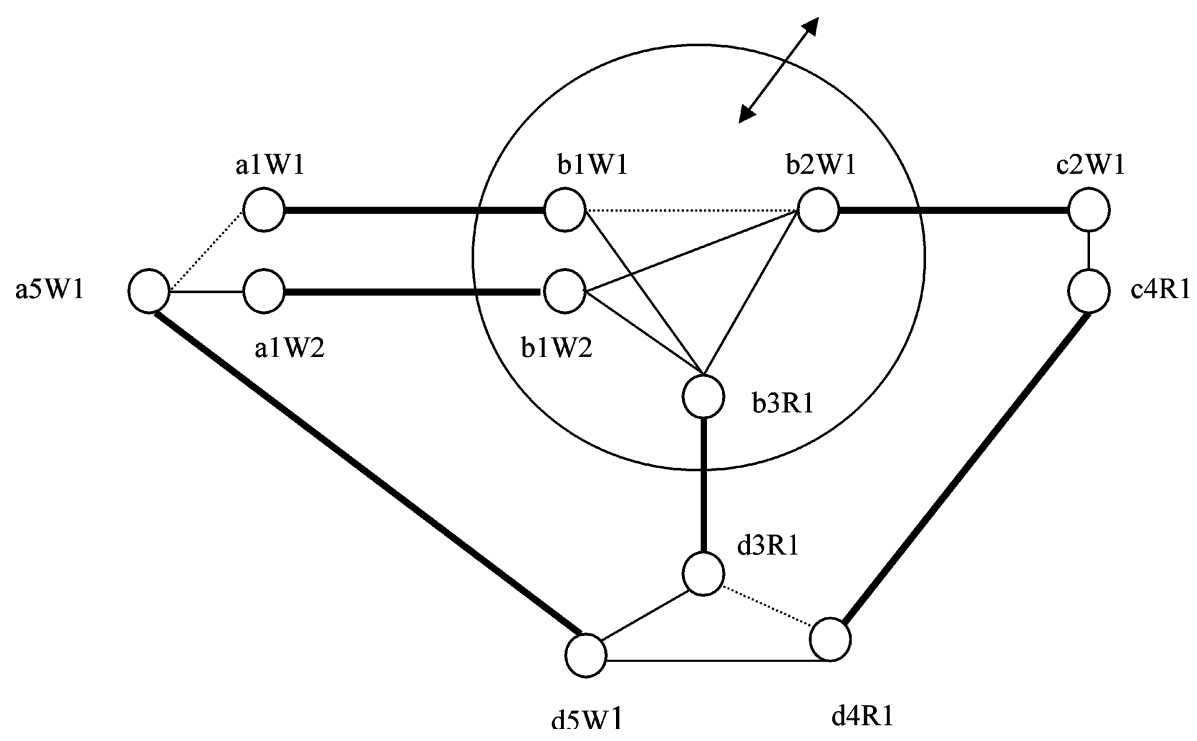

Figure 2: Partial virtual network

way, the example network with multiple modes and means can be represented by a unique but more complex network on which each link corresponds to a unique operation with a specific cost. Obviously, loading and unloading links can also be added at the relevant nodes that correspond to origins and/or destinations. Note that each "virtual node" has a unique label (such as " $b 1 W 1$ "), which is specific to the "virtual network" methodology. A complete discussion of this particular notation can be found in Jourquin and Beuthe [14], but its comprehension is not needed to understand the topics discussed in this paper.

It is important to note that a virtual network makes it possible to assign flows not only between different routes, but also between various transportation modes. Indeed, in opposition to the classical four stages models (generation, distribution, modal-split and assignment), virtual networks, which explicitly decompose all the tasks in the transportation chain, 
combine modal split and assignment in a single step, without any explicit modal-split. A shortest path is such an "operations network" can indeed make use of different transportation modes.

\section{A promising methodology with a hidden trap}

The used cost functions for the different transport operations can be relatively detailed and complex, and for instance take into account the nature of the transported goods. This can lead to the use of different transportation modes for different commodities. Nevertheless, the aggregation level of the origin-destinations matrixes that can be produced for very large areas is such that one cannot guess that, for a given category of commodities, everything is transported by the same transportation mode. Indeed, the demand at the European level for instance is often only available at the NUT1 or NUTS2 level. Even when more sophisticated techniques are used to obtain city to city relations, it is a nonsense to consider that everything that is sent from a given city to another uses the same, unique, transport mode: a factory located nearby a railway station will most probably more often use railway transport than another, located in the same city, but far from the station. Finally, even for a given point to point relation between two factories, some internal logistic considerations make it sometimes useful to use alternative transportation modes.

In the classical models, the modal choice is applied as a separate step. In other words, in such an approach, all the quantities for a given mode are separately assigned on their respective modal networks. In such an approach, each mode can be used on short and long distances with no particular limitations.

However, in "virtual networks", an assignment doesn't search only for a cheapest itinerary, but for a cheapest path that includes all the (un) loading, transit and transhipment operations, so that the simple notion of transportation mode loses a lot of its original meaning, creating a new problem. This will be illustrated by means of a simple example. In Table 1, a small demand matrix is described, that contains data for two transportation modes. The last column is obviously not an input data but a simple computation based on the two previous columns. From this table, it is easy to conclude that 17 tons are transported by mode 1 and 16 by mode 2 . The tons.km for the two modes are respectively equal to 3,850 and 5,400 .

This example clearly illustrates that distance cannot be considered as the only explanatory variable and that there exists some unobserved factors that explain the mode (and route) choice for individual shipments. This is a classical point that has often legitimated

Table 1: Input data for simple example

\begin{tabular}{cccc}
\hline Mode & Distance & Tons & Tons.km \\
\hline 1 & 100 & 4 & 400 \\
1 & 150 & 7 & 1,050 \\
2 & 200 & 6 & 1,200 \\
2 & 300 & 4 & 1,200 \\
1 & 400 & 6 & 2,400 \\
2 & 500 & 6 & 3,000 \\
\hline
\end{tabular}


the use of random utility models to describe actual flows on a network. In passenger transport models, the use of such a stochastic approach is useful because the behaviour of the human being during his journeys can be influenced by a lot of, sometimes subjective, factors. This is particularly true in urban networks. This is less the case for long haul freight transport. However, in large scale models, the details of the network is not sufficient to identify the exact location of each firm and the modal networks it is connected to. Finally, the nature of the demand matrixes is so that, even in the best cases, only information is available from city to city and not from a particular firm to another. All these factors make the demand table 1 , in which the different transport modes are used on different distance classes, an often encountered and plausible scenario.

Consider the two transportation modes have linear cost functions, so that cost $=A+$ $B *$ distance. The values for $\mathrm{A}$ are 2 for mode 1 and 55 for mode 2 . The values for $\mathrm{B}$ are set to 0.5 and 0.3 respectively.

Figure 3 illustrates these two cost functions, and it can clearly be seen that mode 1 will be chosen for the three first elements of the demand matrix, because they are related to distances that are shorter then the break-even distance. Mode 2 will be used for the remaining entries of the matrix. Thus, 17 tons will be assigned to mode 1 and 16 to mode 2 , which are the expected figures. But only 2,650 tons.km will be transported by mode 1 and 6,600 by mode 2 .

The cost functions can obviously be recalibrated in order to try to obtain a better fit in terms of tons.km. For instance, figure 4 illustrates the cost functions when the values of $\mathrm{A}$ and $\mathrm{B}$ are respectively fixed to 52 and 0.35 for mode 2, the values for mode 1 remaining unchanged. In this case, the four first lines of the demand matrix will be assigned to mode 1 , for a total of 3,850 tons.km, which is perfectly right. But now, 21 tons will be transported by mode 1 , which is too much.

This simple example shows that, using a simple all-or-nothing assignment, it is impossible to calibrate an assignment on both the transported quantities (tons) and the flows

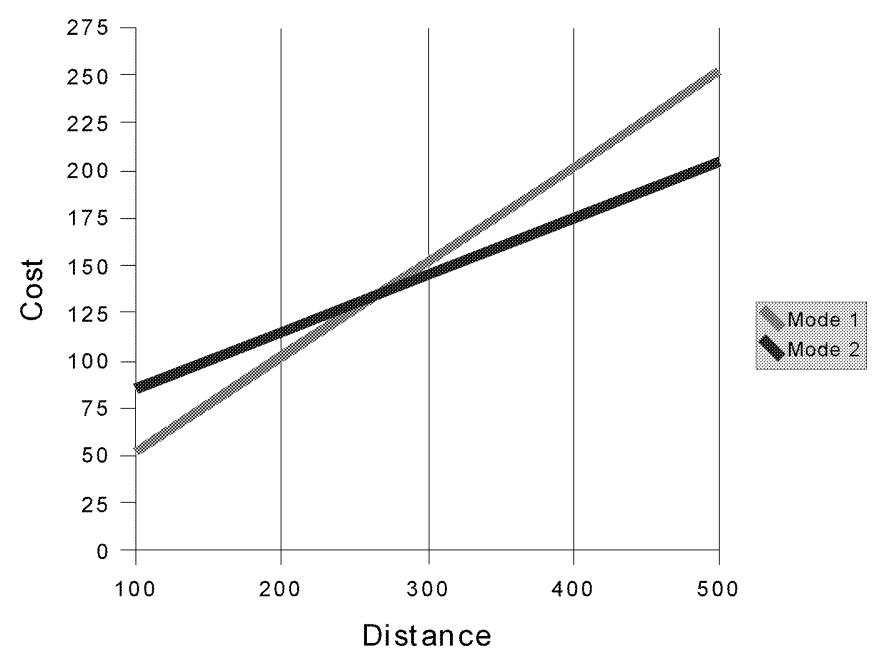

Figure 3 : Original cost functions 


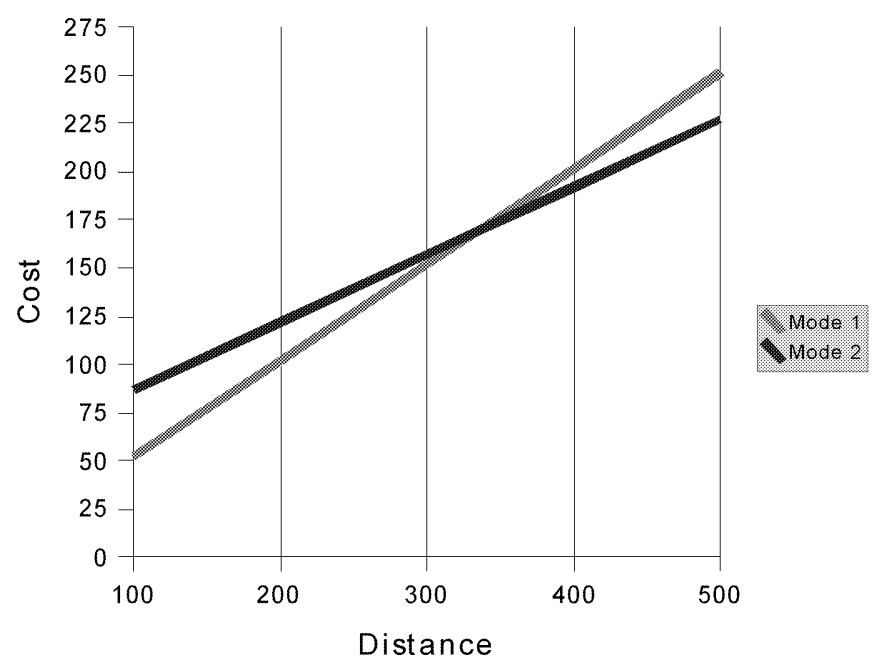

Figure 4 : Modified cost functions

(tons. $\mathrm{km}$ ) when virtual networks are used to avoid separate modal-choice and assignment models. The only way to solve this "distance trap" is to implement technique that doesn't, for a given distance (and origin-destination pair), assign all the demand on the same path (and thus maybe same mode) on a virtual network.

\section{The equilibrium assignment problem and its limits}

A lot has already been written on the assignment problem, and it goes beyond the scope of this paper to present an in-depth review of the existent methods. An assignment problem is the distribution of traffic in a network considering a demand between locations and the transport supply of the network. Assignment methods are looking for a way to model the distribution of traffic in a network according to a set of constraints, notably related to transport capacity, time and cost. This type of problem can be solved using optimization methods. The assignment methods can be grouped in four categories, depending on their ability to take capacity constraints into account or the way they consider the perception the users have of their operating costs.

With the exception of the new "origin-based assignment", developed by Bar-Gera and Boyce [1], which for a given origin considers all the destinations together and optimises the routes serving them ${ }^{1}$, all the equilibrium assignment methods spread the flows over a set of

Table 2: Assignment techniques

\begin{tabular}{|c|c|c|}
\cline { 2 - 3 } \multicolumn{1}{c|}{} & \multicolumn{2}{c|}{ Variable perception of the costs } \\
\hline Capacity constraint & No & Yes \\
\hline No & All or Nothing & Stochastic (multi-flow) \\
\hline Yes & Equilibrium & Stochastic equilibrium \\
\hline
\end{tabular}

\footnotetext{
${ }^{1}$ Unfortunately, this method is not yet enough documented to be easily implemented.
} 
routes obtained by several "All-or-Nothing" (AoN) assignments computed with different costs. The principle of the AoN algorithm is to compute the minimum weight path between each pair of origin and destination, and to allocate the total demand to be transported between these nodes onto this single path.

However, a simple implementation of the AoN algorithm presents some limits because it is often observed that the flow of transport between two given nodes is distributed over various alternative routes. Two main reasons can explain this phenomenon: the capacity constraints of the network and the fact that all the users don't have the same perception of the costs of the different alternative routes. Both reasons induce a spreading of the flow over several routes.

Among the possible alternatives, the equilibrium algorithms take into account the variation of the transportation costs according to the assigned flow, considering that the distribution of the traffic over the network is the result of an interaction between the supply and the demand for transport.

As outlined earlier, the implementation of these methods on a virtual network makes it possible to observe transfers of flow not only between different routes, but also between various transportation modes, which is an interesting feature that could be helpful to solve the "distance trap".

The equilibrium assignment models require cost functions which are related to the flow $\mathrm{V}$ on the network. Such a relation, for a given link a, can be generally expressed as : $C_{a}=$ $C_{a}(\{V\})$. Many functions were proposed to describe this relation. Ortúzar and Willumsen [17] give a good overview of them.

Various assignment algorithms, which try to obtain an equilibrium distribution of flows on the network, can be found in the literature. The implementation of these algorithms on virtual networks is not immediate. Indeed, congestion is observed on real links, not on virtual links. In a virtual network, the same real link is represented by various virtual links, according to the number of transportation means (types of vehicles) that are defined. It is thus necessary to consolidate the flows obtained on the virtual links related to a same real link in order to obtain the total flow on the real link.

It obviously goes beyond the scope of this paper to give an in-depth description of the most used equilibrium assignment techniques. The well known method of successive averages (MSA, Smock [19]) and Frank-Wolfe (FW, Frank and Wolfe [7]) were adapted to virtual networks.

The incremental (INC) assignment, which is not a real equilibrium technique was also implemented. The main disadvantage of the incremental method is that once a flow is assigned to a link, it is not anymore possible to withdraw (a part of) it in order to assign it to another link. Consequently, this method will not necessary ensure that the algorithm converges to an equilibrium solution as in MSA and FW (Sheffi [18]). Note that in order to accelerate the convergence of the algorithm, the incremental method can also be associated with the Frank-Wolfe's algorithm to obtain the initial flows (Inc $+\mathrm{FW}$ ). However, this method can be counterproductive, especially if the network is not congested.

All these algorithms were tested on the extensive trans-European multi-modal network, as defined by the European Conference of Ministers of Transport. The digitised network 
that what used contains about 17.000 links and 12000 nodes, from which about 2000 are potential origins and/or destinations. The implementation of the capacity restrained methods is not immediate, because the roads are shared by private cars and trucks. To give a reasonable answer to this problem, the capacity of the roads was fixed to a theoretical residual capacity that is left over when the private cars are already on the network.

However, since the O-D matrixes ${ }^{2}$ contain annual amounts of goods on inter-urban and inter-regional and international relations, the use of equilibrium assignment techniques raises a certain number of questions. Indeed, the nature of the demand matrixes is quite different from urban $\mathrm{O}-\mathrm{D}$ matrixes set-up for peak hours, because no information is available on the departure and/or arrival time of the commodities. Moreover, as distances are long, a single trip often occurs during different periods of the day, including peak hours.

In order to try to stress the model, the assignments were performed in a scenario in which the passenger traffic during an average peak period was estimated. To be simple, the available capacity on the road network for freight transport was set to the actual capacity of the road chunks from which the estimates passenger flows during the peak periods were withdrawn. No capacity restrictions were introduced for rail or waterborne transports, i.e., their respective cost functions are not dependent on the already assigned flows.

Once the residual capacities on the network and the O-D matrixes for an average peak hour are known, the different assignment methods were tested. The results are shown in Figure 5. It is worthwhile to note that the found solution is only slightly more expensive $(+1 \%)$ than the initial AoN solution. Several factors can explain this:

- The used O-D matrixes contain total quantities on an annual basis, and it is difficult to estimate what is transported at a given time of a day.

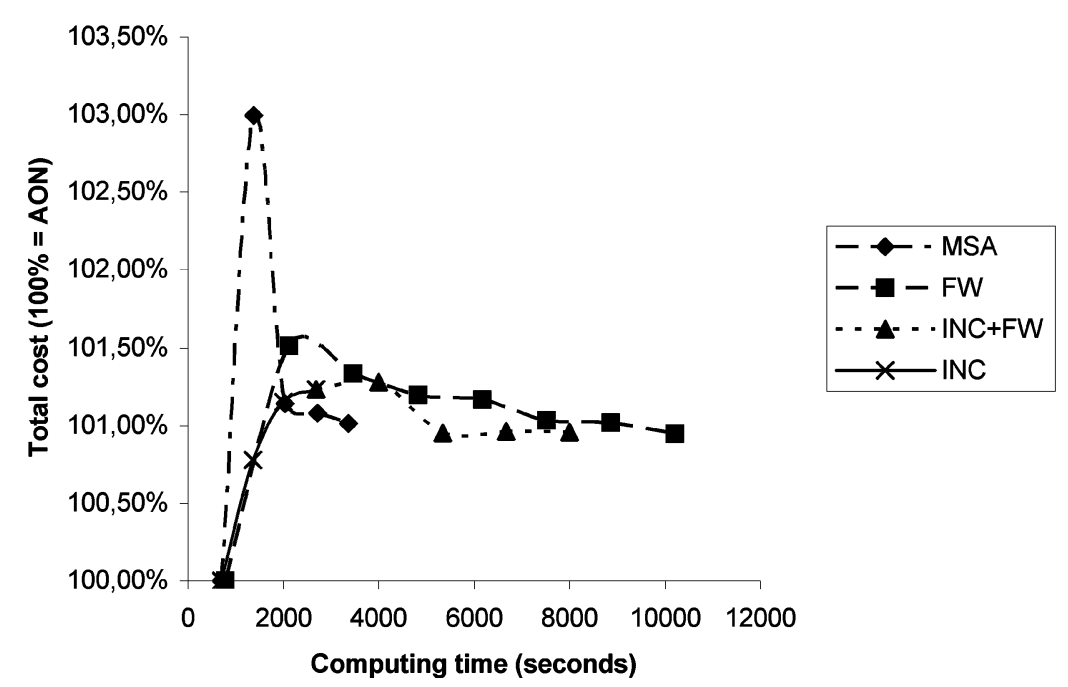

Figure 5: Equilibrium assignment results

\footnotetext{
2 The used matrixes are thoroughly discussed in Geerts and Jourquin [8].
} 
- The implemented assignment algorithms are "static" and the sense that the vehicles are not tracked during their journey. This is a problem for the assignment on long distances, because often, several hours or even days (including peak and off-peak periods) pass between the starting and the arrival time. The assignments don't take into account the actual location of the vehicles at a given time.

- Congestion is mostly observed in and around urban areas. The transportation networks that are located in such areas only represent a small part of the total transEuropean network that is used in this exercise.

Finally, these assignment methods applied on "virtual networks" only have a limited impact on the market-share of the different transportation modes, as shown by Table 3 .

The equilibrium methods were implemented in order to try to ensure that the total flow to assign between a given origin and destination is spread over several routes (and thus modes if virtual networks are used). Unfortunately, it appears that capacity restrictions alone are not an explanatory factor that is strong enough to reach the expected result, for all the reasons that were just outlined. In other words, it becomes clear that equilibrium assignment techniques don't give an adequate solution to the "distance trap" and, therefore, other approaches must be explored.

Table 3 : Impact on the estimated modal split

\begin{tabular}{|c|c|c|c|}
\hline & \multicolumn{3}{|c|}{ Variation of modal split induced by equilibrium solutions } \\
\hline Method & Road & Rail & Water \\
\hline MSA & $-2.56 \%$ & $+0.80 \%$ & $+5.75 \%$ \\
\hline FW & $-3.23 \%$ & $+1.15 \%$ & $+7.09 \%$ \\
\hline Inc+FW & $-3.34 \%$ & $+1.16 \%$ & $+7.16 \%$ \\
\hline Inc $(\mathbf{n}=\mathbf{4})$ & $-1.62 \%$ & $+0.35 \%$ & $+3.64 \%$ \\
\hline
\end{tabular}

\section{The multi-flow assignment problem on virtual networks}

Equilibrium assignment techniques, used on inter-urban, regional or international freight networks are not able to enough reflect the modal and route behaviours. Other deterministic or stochastic multi-flow algorithms must be implemented and tested on virtual networks. In other words, the capacity restrictions are certainly not the only criteria that lead to the choice of a given route and/or transportation mode.

Identifying the choice set in a route choice context is a difficult task. Two main approaches can be considered.

First, it may be assumed that each individual can potentially choose any path between her/his origin and destination. The choice set is easy to identify, but the number of alternatives can be very large, causing operational problems in estimating and applying the model. Moreover, this assumption is behaviourally unrealistic for freight transport.

Second, a restricted number of paths may be considered in the choice set. The choice set generation can be deterministic or stochastic, depending on the analyst's knowledge of the 
problem.

Dial [5] proposes to include in the choice set "reasonable" paths composed of links that would not move the traveller farther away from her/his destination. The labelling approach (Ben-Akiva et al. [2]) includes paths meeting specific criteria, such as shortest paths, fastest paths, most scenic paths, paths with fewest stop lights, paths with least congestion, paths with greatest portion of freeways, paths with no left turns, etc. This needs a lot of detailed information that is often not available when large areas, such as a country or a continent are to be covered.

Thus, in order to implement multi-flow assignments, a pragmatic method must be proposed to compute a set of realistic alternative routes, over which the flow can be spread. This is done by the so called multi-path algorithms. The K-shortest paths problem ranks the $\mathrm{K}$ paths connecting a given source-destination pair in the network with minimum total cost. The first algorithm for solving this problem was proposed by Hoffman and Pavley [12], and other algorithms have been proposed later. The Martins et al. [16] algorithm is currently the most effective solution in terms of running time complexity. However these algorithms appear to produce a set of paths that are not very useful. Indeed, the next best path often differs very few from the original path. A classical example is to leave a highway for only a few hundred meters before to take it again. These overlapping two routes obviously cannot be considered as valid alternatives.

To solve this, we have adopted another strategy to compute a set of alternative paths : once a shortest path has been computed, the costs on all the links on this path are artificially increased before a next best path is computed. This pragmatic solution can be outlined as :

1. Compute the cheapest paths

2. Increase the cost on each link that belongs to this and the previous computed paths.

3. Iterate. Go to Step 1

Such an implementation is quite simple and ensures that, because the costs on the used links are gradually increased, each new route will try to avoid already used links. However, the most time-efficient implementation, in which the algorithm of Dijkstra [6] is used to compute the shortest paths, can lead to inaccurate results, due to the fact that this algorithm not only computes the shortest path between an origin and a destination, but a tree that represents the shortest paths from a given origin to all the possible destinations. This can be illustrated by the following example.

In Figure 6, the figures on the links represent their respective costs. If the algorithm of Dijkstra is used to compute the shortest paths starting from node $A$, the obtained result will be the one illustrated by the bold lines, as Dijkstra computes all the paths from a given origin to all the possible destinations. If the above described algorithm is used to compute the second best routes, the costs on the already used links must be increased, for example by adding $50 \%$ to the cost of these links. Figure 7 , which is focused only on the $A$ to $C$ route, illustrates this but also shows that, if a new shortest route is now computed between $A$ and $C$, it will use the two links that have a real (initial) cost of 7 . However, the second best route between $A$ and $C$ that could be computed on the original network represented by figure 


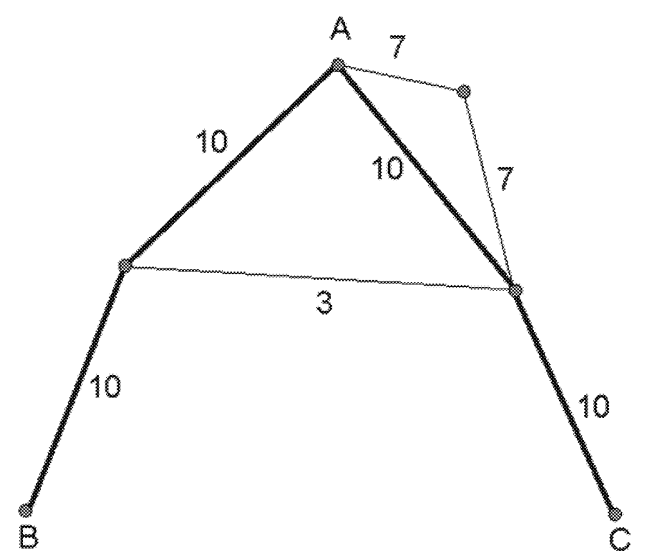

Figure 6: A Dijkstra tree computed from node A

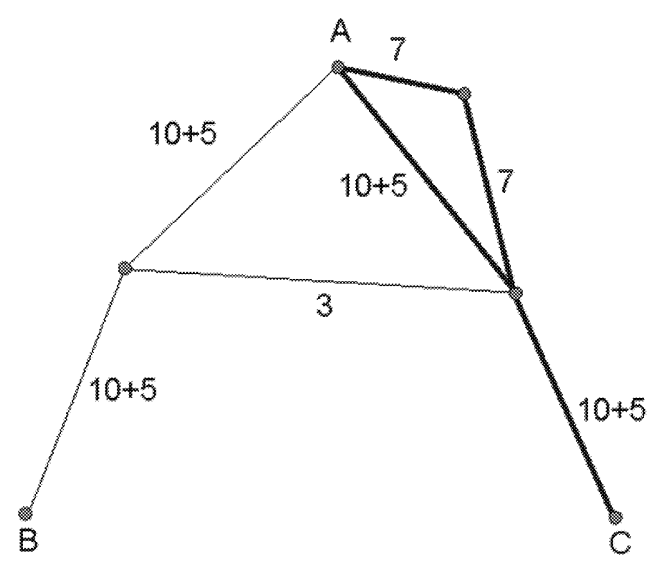

Figure 7 : Inexact set of $\mathrm{A}$ to $\mathrm{C}$ paths

6 is expected to be the one that starts from $A$ in direction of $B$, then going to $C$ at the first crossroad. This will not be found just because the first section has now a cost of 15 , because it was used on the initial path from $A$ to $B$. In other words, using Dijkstra, the resulting second best itinerary between an origin and a destination can be influenced by the other already computed routes starting from the same origin and going to other destinations.

A way to avoid this is to use a "point to point" algorithm to compute the shortest path. The most efficient one is the $A$ *algorithm (Hart et al. [10 \& 11]), but the assignment using this algorithm is much more slower because it must be applied on each cell of the origindestination matrix, when the algorithm of Dijkstra is only called once for each line of the matrix. Table 4 gives the computing time needed for the different types of assignments of a matrix that contains about 250,000 cells on a virtual network generated from the digitised trans-European network. The computation times were obtained on a Pentium 4 3.4Ghz HT computer running Linux Fedora Core 4 with a 2.6.14 SMP kernel. The algorithms were implemented and profiled in our Nodus ${ }^{\mathrm{tm}}$ software package, written in Java 1.5. Nodus (http://www.fucam.ac.be/nodus) can be used for many different analytical tasks and for 
Table 4 : Assignment performances

\begin{tabular}{|l|c|}
\hline \multicolumn{1}{|c|}{ Assignment method } & Computation time \\
\hline All or Nothing & $5 \mathrm{~min}$ \\
\hline Fast multi-flow ${ }^{3}$ (Dijkstra) & $15 \mathrm{~min}$ \\
\hline Exact multi-flow $\left(A^{*}\right)$ & 12 hours \\
\hline
\end{tabular}

optimizing various objective functions, but, until now, it has been used mainly for analysing minimum cost solutions of transport flows over extensive international networks. Actually, our initial motivation was to develop a tool for evaluating the economic impacts of new transport infrastructures and policies, of which cost savings constitute an important benefit of new infrastructure. With this model, they can be easily computed in a comprehensive way, taking into account all networks effects. Furthermore, it can estimate various direct impacts, which can be linked to transport operations, such as energy consumption, labour cost or the external costs of pollution and congestion, which make it an appropriate tool for travel demand control.

The exact algorithm really needs a lot of time. So, the question of the pertinence of its use can be raised. A definitive answer to this question cannot be given, and will probably be influenced by the nature of the problem to solve. To give an idea, using the just outlined OD matrix and map, the amount of tons transported by the dominant mode (road) doesn't vary with the multi-flow procedure and the tons.km change by $2 \%$. The same variation $(2 \%)$ is observed on the total cost on the system.

Whatever the shortest-path algorithm chosen, a set of alternative paths can be obtained. But will this set give an adequate answer to the "distance trap" problem identified earlier in this paper?

The answer is unfortunately negative in most cases because, on virtual networks, the second best itinerary often uses the same transportation mode that the best one. Indeed, the difference in costs for two different transportation modes between a given OD pair is often larger than the difference between the cheapest path and the second best one.

Our algorithm can therefore be somewhat refined to solve the problem:

1. Compute the cheapest path

2. Increase the cost on each link that belongs to this and the previous paths.

3. Increase the cost for each loading operation at the origin node (i.e. in every possible direction starting from the node) for the transportation mode used on the first link of the path.

4. Iterate. Go to Step 1

Now, not only the costs on the used itinerary are changed, but also the costs of the initial loading on the chosen transportation mode are increased on all the directions that start from the initial node meaning that, whatever alternative route will be computed during the next

\footnotetext{
${ }^{3}$ The multi-flow algorithms were implemented to compute three alternative paths.
} 
iteration, the use of the same transportation mode will be discouraged.

The results that can be obtained are clearly illustrated by figure 8 . The map at the topleft represents a simple network on which roads and railways are present. At the top right, the unique path that is displayed is a result of an all-or-nothing assignment. The two maps on the bottom row clearly show the difference between a "simple" and a "forced" multi-flow assignment (here computed for two alternative paths).

We now can generate a set of credible alternative paths that also includes, were possible, a set of alternative transportation modes. In other words, a modal choice is available for all the entries of the demand matrixes, regardless of the distance between the origins and the destinations. This can be considered as a satisfactory answer to the "distance trap" discussed earlier, because there is now a possibility, for each origin-destination pair, to spread the flow over several routes and modes, which was not the case when equilibrium algorithms were implemented.

The last step that must be performed consists in spreading the demand over the different identified paths. The route choice model is a central component of spatially disaggregate models and can be either deterministic or stochastic. In the deterministic case, it is assumed that drivers select the path that minimizes the total generalized costs to their destination. When there is network congestion, this path does not coincide, in general, with the path that minimizes distance: drivers are diverted to longer routes but that are less congested. This approach and its limits have been described in the previous section.

Another critic that is often found in the literature is that the deterministic route choice is not behaviorally sound when drivers are faced with quasi identical alternatives, and it can be assumed that both routes will be selected with almost equal probability. Moreover, drivers have limited cognitive abilities, so that it is unrealistic to assume that they always select the best path in a multi-thousand link network. This is known as the perceived cost and introduces the motivation for the stochastic models.

Historically, many models have been used to represent the situation in which traffic diverts from an old, overloaded route, to a new, faster but longer route. These models are referred to as binary route choice models, under which falls the well known binary Probit model. The Probit model assumes that the random error term of the distribution of the perceived costs has a normal distribution. If these error terms have independent and identically distributed Weibull distribution, the model is known as a binary Logit model.

Multiple route choice models differ not only in the error distribution on which they are based, but also because they assume different definitions of acceptable routes and evaluate routes in different ways. As far as the error distributions are concerned, it is generally the case that the extension from two to many routes results in a model in which the probabilities of the usage of these routes cannot be estimated exactly. An exception to this, and even then only on the basis of very restrictive conditions, is the extension of the binary Logit model, which may be extended to the multiple choices: the Multinomial Logit Model (MNL). However, the MNL models have a limitation for some practical applications, often illustrated by the red bus/blue bus paradox (see, for example, Ben-Akiva and Lerman [3]) in the modal choice context. This limit is linked to the fact that alternative routes which share a large amount of common chunks are often chosen because of the hypothesis made on the 


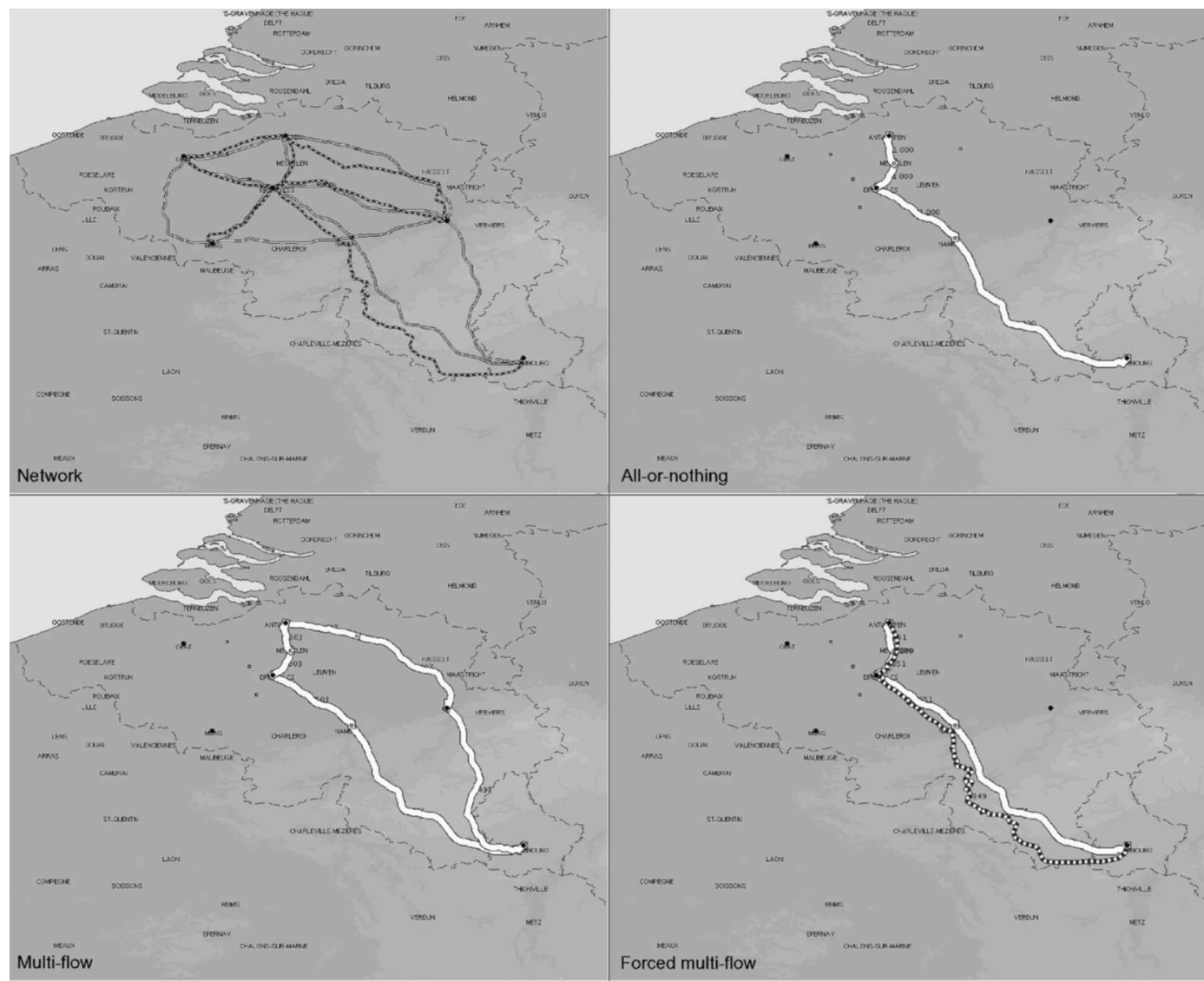

Figure 8: Sample assignments between two nodes

independence of the error terms. However, the way we have computed the set of alternative routes may give an interesting answer to this problem, as the routes should be "really" different. The Nested Logit Model, partly overcomes this limitation of the Multinomial Logit Model, but cannot be applied to paths on virtual networks, because "categories" (in this case transportation modes) of paths should be identifiable in the path. This is not the case because a path is a finite suite of transport operations, including the use of one or more transportation means. In other words, the simple notion of "mode" disappears somewhat.

Thus, the MNL model seems to be suitable for our problem. It can classically be formulated as ;

$$
P_{i}=\frac{\exp \left(\beta C_{i}\right)}{\sum_{j \in K} \exp \left(\beta C_{j}\right)}
$$

Where

- $P_{i}$ is the probability to chose path $i$ among $K$ alternatives;

- $C_{i}$ the cost on path $i$.

There is no generally accepted value for $\operatorname{parameter} \beta$, which must be estimated using 


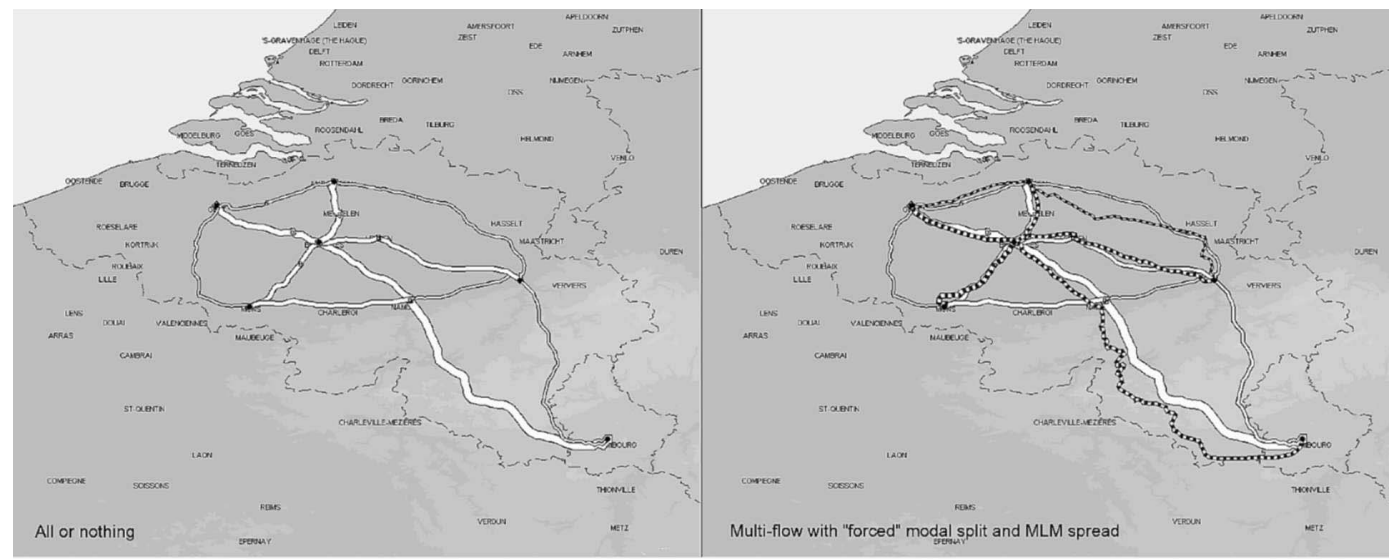

Figure 9: Sample assignments of a OD matrix

collected data or revealed preference studies. If this cannot be done, one could also argue that the flow could be spread over the different alternatives paths according to their relative weight. This is very simple and pragmatic, but can however be justified for strategic models when generalised cost functions, that include time and quality related aspects are used for the different transport operations that are decomposed in the virtual network. If this latest option is chosen, the multi-flow assignment becomes purely deterministic. Of course, $\beta$ can also be calibrated to represent the observed dispersion of choices. Whatever spreading method is used, the assignment on a virtual network will produce, in a single step, a result that combines the use of different transportation modes on a same point-to-point relation, which is obviously very different from what can be obtained by an all-or-nothing (or even equilibrium) method. This is clearly illustrated by figure 9 .

\section{Conclusions}

Transportation models are often based on the classical four stages approach, in which generation, distribution, modal-split and assignment are seen as separated steps. Another approach, promoted by different authors, is to create an alternative network, that not only represents the geographic components of the network, but also the different operations that are possible during the transportation chain. These "super networks" or "virtual networks" open interesting perspectives, because both the modal choice and the assignments steps of the classical approach are performed at the same time.

Unfortunately, these virtual networks contain a hidden trap, as its nearby impossible to calibrated the models on both the transported quantities and the flows (expressed in tons. $\mathrm{km})$. This problem which is referred to in this paper as the "distance trap" can only be solved if the flow that must be sent from an origin point to some destination is spread over several routes and transportation modes.

It appears that the use of equilibrium assignment procedures don't give an adequate answer on large scale networks such as the trans-European multi-modal freight network. 
This is essentially due to the fact that equilibrium models are only efficient at a local level, and were congestion, or at least heavy flows, are observed. Now, origin-destinations matrixes for long distance transport are often built on a yearly basis only, and it is thus difficult to estimate what happens during the peak hours. Even more problematic is the fact that long distance transport last several hours, or even days, and it is not possible, with static models, to know were a vehicle is located at a given moment.

Obtaining a set of credible alternative routes, i.e. (nearby) non overlapping routes, is only possible if multi-flow algorithms are used. It becomes rapidly clear the K-shortest paths algorithms don't offer a satisfactory solution, because the obtained routes are almost the same. A simple and pragmatic algorithm is proposed that ensures that the computed set of paths contains both different itineraries and the use of different transportations modes, which is the goal to reach in order to find a solution to the "distance trap".

Finally, the methods to use to spread the flow over the different routes are discussed. It seems that the Multinomial Logit is the best alternative. A very pragmatic solution using the relative weights of the different paths in the set of alternative routes could also be justified if the MLM cannot be calibrated due to a lack of collected information.

\section{References}

[1] Bar-Gera, H. and Boyce, D., "Origin-based algorithms for combined travel forecasting models", Transportation Research B, Vol. 37, No. 5, 2003, pp. 405-422.

[2 ] Ben-Akiva, M., Bergman, M. J., Daly, A. J. and Ramaswamy, R., "Modelling Inter-Urban Route Choice Behavior", Proceedings of the 9th International Symposium on Transportation and Traffic Theory, 1984, pp. 299-330.

[3 ] Ben-Akiva, M. and Lerman, S. R., Discrete Choice Analysis, Cambridge, MIT Press, 1985.

[4] Crainic, T.G., Florian, M., Guélat, J., and Spiess, H., "Strategic planning of freight transportation : Stan, an interactive graphic system", Transportation research record, 1283, 1990, pp. 97-124.

[5] Dial, R. B., "A probabilistic multipath traffic assignment model which obviates path enumeration”, Transportation Research, Vol. 5, 1971, pp. 83-111.

[6 ] Dijkstra, E.W., "A note on two problems in connection with graphs", Numerische Mathematik, Vol. 1, 1959, pp. 269-271.

[ 7 ] Frank, M. and Wolfe, P., "An algorithm for quadratic programming”, Naval Research Logistics Quarterly, Vol. 3 , 1956, pp. 95-110.

[8] Geerts, J.F. and Jourquin, B., "Freight Transportation Planning on the European Multimodal Network: the case of the Walloon Region", European Journal for Transport Infrastructure Research, Vol. 1, No. 1, 2001, pp. 99-106.

[9] Harker, P.T., Predicting intercity freight flows, VNU Science Press, 1987.

[10] Hart, P. E., Nilsson, N. J. and Raphael, B., "A Formal Basis for the Heuristic Determination of Minimum Cost Paths", IEEE Transactions on Systems Science and Cybernetics SSC4 Vol. 2, 1968, pp. $100-107$.

[11] Hart, P. E., Nilsson, N. J. and Raphael, B., "Correction to "A Formal Basis for the Heuristic Determination of Minimum Cost Paths", SIGART Newsletter, 37, 1972, pp. 28-29.

[12] Hoffman, R. and Pavley, R.R., "A method for the solution of the $\mathrm{n}^{\mathrm{th}}$ best path problem", Journal of the association for Computing Machinery, Vol. 6, 1959, pp. 506-514.

[13] Jourquin, B., Un outil d'analyse économique des transports de marchandises sur des réseaux multi- 
modaux et multi-produits: Le réseau virtuel, concepts, méthodes et applications, PhD Thesis, Facultés Universitaires Catholiques de Mons, Belgium, 1995.

[14] Jourquin, B. and Beuthe, M., "Transportation policy analysis with a geographic information system : the virtual network of freight transportation in Europe", Transportation Research C, Vol. 4, No. 6, 1996, pp. 359-371.

[15] Jourquin, B. and Limbourg, S., “Assignment techniques on Virtual Networks, Performance considerations on large multi-modal networks", European Regional Science Association congress (ERSA) 2003, University of Jyväskylä - Finland, August 2003.

[16] Martins, E.Q.V., Pascoal, M.M.B. and Santos, J.L.E., “A new improvement for a k shortest paths algorithm”, Investigação Operacional, 21 (1), 2001, pp. 47-60.

[17] Ortúzar, J. de D. and Willumsen, L., Modelling Transport, John Wiley \& Sons, New-York, 1990.

[18] Sheffi, Y., Urban Transportation Networks : Equilibrium Analysis with Mathematical Programming Methods, Prentice-Hall, Englewood Cliffs, New Jersey, 1985.

[19] Smock, R.J., "An iterative assignment approach to capacity restraint on arterial networks", Highway Research Board Bulletin, Vol. 156, 1962, pp. 1-13. 